\title{
Development of a research reactor power measurement system using cherenkov radiation
}

\author{
Brício Mares Salles ${ }^{1}$, Amir Zacarias Mesquita ${ }^{1 *}$, and Marley Rosa Luciano ${ }^{2}$ \\ Center for the Development of Nuclear Technology, Campus of UFMG/Pampulha, Belo Horizonte, MG, Brazil ${ }^{1}$ \\ Newton Paiva University Center, Belo Horizonte, Minas Gerais, Brazil ${ }^{2}$
}

\section{(C)2018 ACCENTS}

\begin{abstract}
Nuclear research reactors are often found in open pools, allowing visibility of the core and the bluish luminosity of the Cherenkov radiation. In general, the thermal energy released in these reactors is monitored by chambers that measure neutron flux, because this is proportional to the power. There are other methods used to measure the power, including measure of the fuel rod central temperature and the energy balance in the heat exchanger. The brightness of Cherenkov radiation is caused by the emission of visible electromagnetic radiation (in the blue band) by charged particles that pass through an insulating medium (water in nuclear research reactors) at a speed greater than that of light in this medium. This effect was characterized by Pavel Cherenkov, earning him the Nobel Prize in physics in 1958. The objective of the present project is to develop an innovative and alternative method to monitor the power of nuclear research reactors. This will be done by analyzing and monitoring the intensity of luminosity generated by the Cherenkov radiation in the reactor core. This method will be valid for powers up to $250 \mathrm{~kW}$, because above this value the brightness is saturated, as determined by previous studies. The reactor that will be used to test the method is the IPR RI Triga, located at the Nuclear Technology Development Center (CDTN), currently with a maximum operating power of $250 \mathrm{~kW}$. This project complies with the recommendations of the International Atomic Energy Agency (IAEA) on the safety of reactors. It will provide more redundancy and diversification in this measurement and will not interfere with the operation of the reactor.
\end{abstract}

\section{Keywords}

Cherenkov radiation, Nuclear reactor power, Reactor safety, Triga research reactor.

\section{Introduction}

In any nuclear installation, the main concern is safety. The IAEA states that the fundamental objective of safety is to protect people and the environment from the harmful effects of ionizing radiation. They suggest ten safety principles that must be followed. The IAEA recommendations focus on the concepts of redundancy, diversity and independence; in other words, there must be more than one device, with different operating principles, completely independently performing the same function [1]. Safety begins with controlling the parameters and variables in a nuclear reactor; the primary one is the power, which allows the determination of other relevant factors.

*Author for correspondence

This work was supported by Nuclear Technology Development Centre (CDTN), and Brazilian Nuclear Energy Commission (Cnen).
In power measurements, redundancy and diversity are critical, and accuracy and reliability are very important. For this reason, nuclear reactors use several devices to measure the power of the core [2].

In the research reactor IPR-R1 Triga (Figure 1 and Figure 2) (which will be used for the tests proposed in this document) four devices are used to determine the core power, including neutron-sensitive cameras, a fission chamber, two compensated ionization chambers and an uncompensated ionization chamber [3]. This research reactor is located in the Nuclear Technology Development Center (CDTN). It is an open pool-type reactor and the fuel is cooled by natural convection. The system was updated in the $1970 \mathrm{~s}$ to reach up $100 \mathrm{~kW}$. Later, it was updated again to its current system, which allows it to reach $250 \mathrm{~kW}$ in the steady-state. 


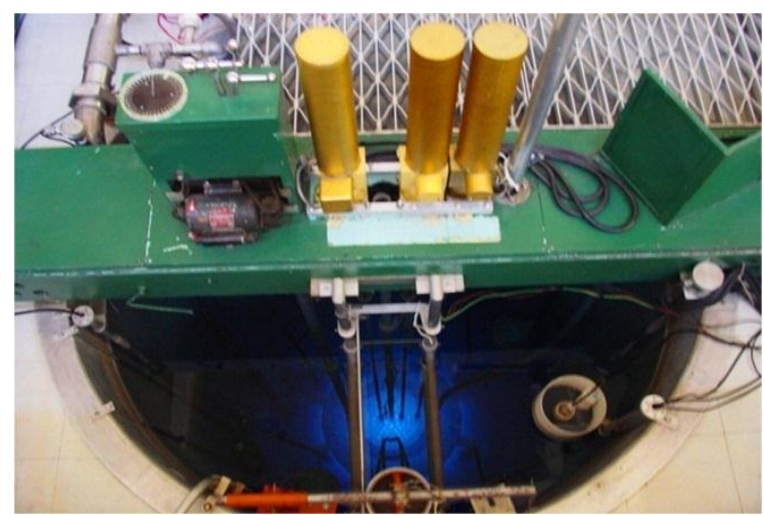

Figure 1 Pool and the core with the research reactor IPR-R1 Triga reactor in operation [3]

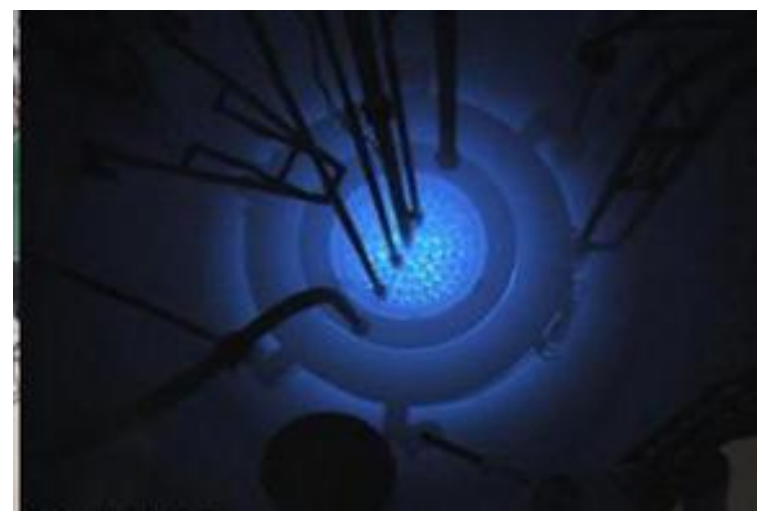

Figure 2 Cherenkov radiation around the core of reactor IPR-R1 Triga [3]

Triga reactors are the most popular research reactors in the world. There are more than sixty facilities operating in several countries [4]. Their popularity derives from the fact that they are the only research reactors that can provide true inherent safety, in addition to the usual engineering safety. This is possible due to the properties of the fuel: uranium hydride and zirconium provide unparalleled safety features, allowing flexibility in settings, with minimal environmental effects [5].

The objective of the project was to develop an innovative and alternative method to control the power of nuclear research reactors. This will be done by analyzing and controlling the intensity of luminescence generated by the Cherenkov radiation in the reactor core. This method will be valid for power up to $250 \mathrm{~kW}$, because above this value, the brightness becomes saturated, as determined by previous studies [6].

\section{Cherenkov radiation}

Electromagnetic radiation known as Cherenkov light is emitted when a charged particle moves in a dielectric medium at a speed greater than the speed of light for that medium. A conspicuous example of that effect is the characteristic blue glow of a pool-type reactor.

Cherenkov radiation is produced through a number of ways when: (a) beta particles emitted by fission products travel with speeds greater than the speed of light in water and (b) indirect ionization by Gamma radiation produces electrons due to photo electric effect, Compton effect and pair production effect. Among these electrons, Compton electrons are the main contributors to Cherenkov radiation [6]. Compton scattering of gamma rays and its intensity is linearly related to reactor fission power, and can be transmitted from the source at the reactor core to a sensing device by means of a highly reflective metallic tube [7].

According to classical physics, a moving charged particle emits electromagnetic waves. In a quantum mechanical picture, when a charged particle moves inside a polarizable medium with molecules, it excites the molecules to the higher levels and excited states. Upon returning back to their ground state, the molecules re-emit some photons in the form of electromagnetic radiation. According to the Huygens principle, the emitted waves move out spherically at the phase velocity of the medium. If the particle motion is slow, the radiated waves bunch up slightly in the direction of motion, but they do not cross. However if the particle moves faster than the light speed, the emitted waves add up constructively leading to a coherent radiation at angle $\theta$ with respect to the particle direction, known as Cherenkov radiation. The signature of the effect is a cone of emission in the direction of particle motion [8].

\section{Materials and methods}

It is intended to perform experiments in the Triga IPR-R1 reactor for the validation of the assembled instrument. Thus, the final adjustments will be made to the luminance to power conversion equations.

The equations will be established after the tests in the Triga reactor, where the luminance values measured by the device will be recorded for each $\mathrm{kW}$ varied in the reactor power. The power variation will be observed by the neutron measuring channels of the control console. 
In order to measure the power of the reactor, there are two outstanding proposals that use photo-diodes, (as the Tehran researchers Arkani and Gharib (2009) [6], do in a similar fashion), and use microcontrollers (such as those of Arduino) with compatible brightness sensors.

\subsection{Proposal for photodiodes}

In this configuration, the photodiodes (Figure 3) will be organized in a two-dimensional polar matrix, to amplify the absorption range of the light. When the Cherenkov radiation reaches the diode, it creates a free electron and a positive "hole" in the depletion region. Moving the holes towards the anode, and the electrons towards the cathode, produces a photocurrent [6]. This "sensor" is connected to a signal amplifier and conditioner that will transport the output signal to a data acquisition system, to calculate the power of the core through the luminosity readings and display them on a screen. A block diagram for this proposal is displayed in Figure 4.

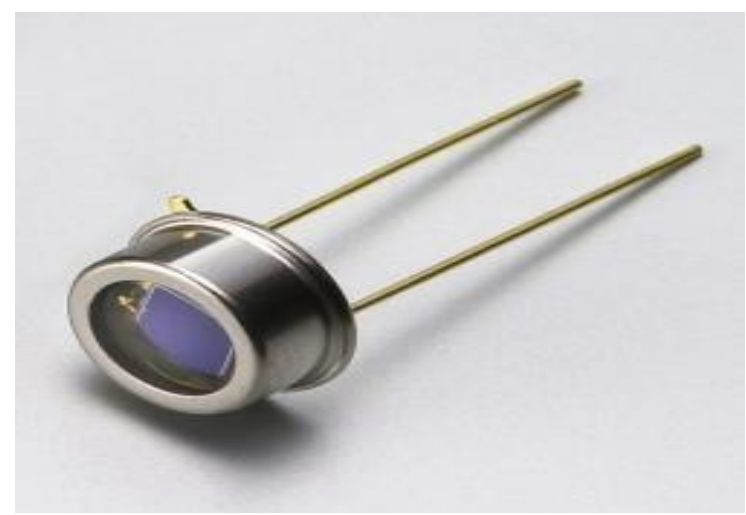

Figure 3 Photodiode [9]

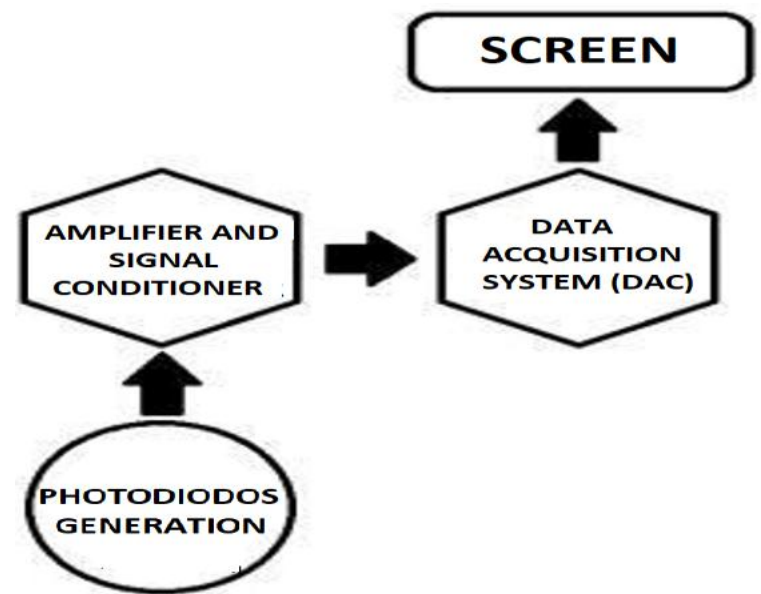

Figure 4 Block diagram for the proposal of the photodiode

\subsection{Microcontroller proposal}

For this configuration, an Arduino board (Figure 5) will be used, with a brightness or color sensor attached, to be chosen by testing the models available on the market. Core power will be calculated by an algorithm and presented in a display unit that can be a computer screen, a smartphone or a specific screen for the device. The first prototypes will be built with the digital brightness sensor TSL2561 (Figure 6) and the color sensor TCS3200 (Figure 7), since they are the most popular sensors for acquiring light data. A block diagram for this proposal is presented in Figure 8 .

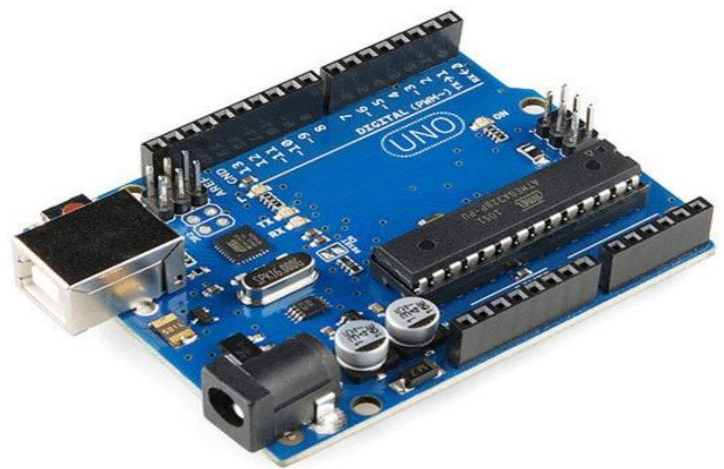

Figure 5 Arduino UNO board [10]

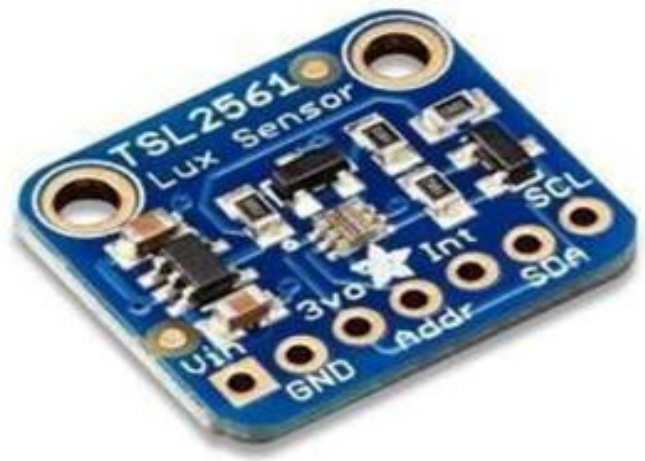

Figure 6 TSL2561 digital luminosity sensor [11]

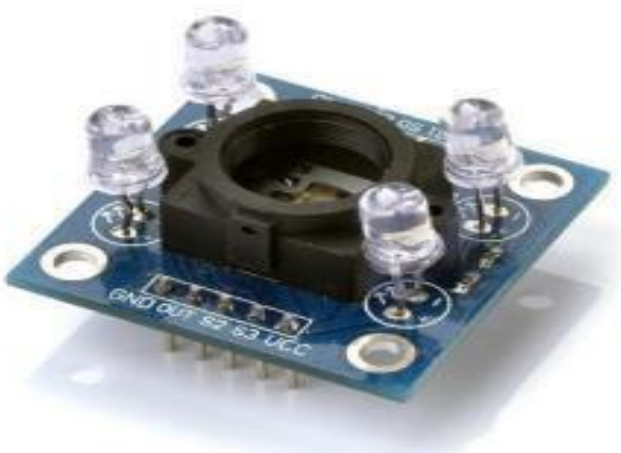

Figure 7 TCS3200 colour sensor [12] 

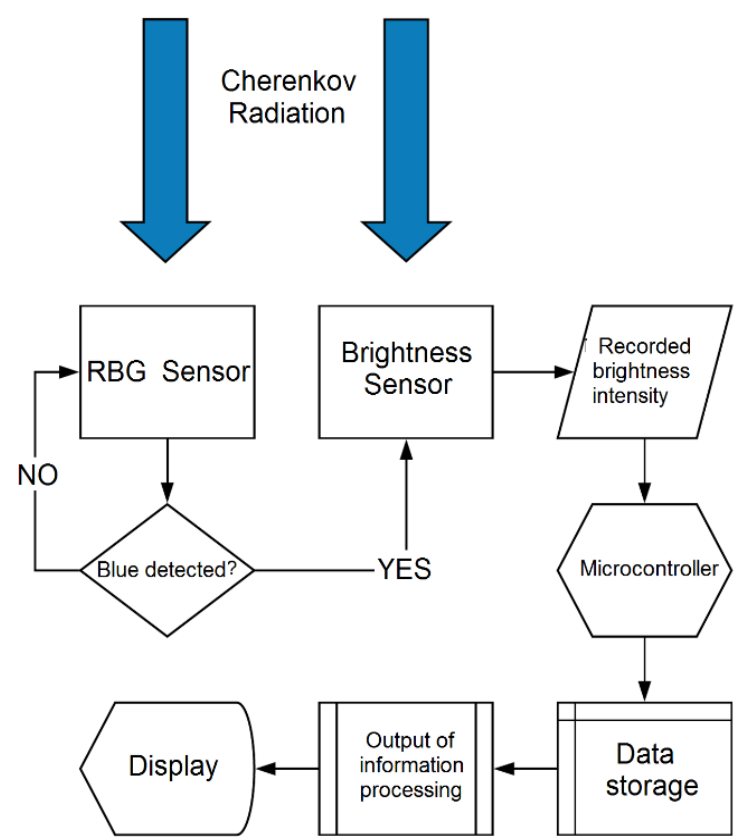

Figure 8 Block diagram of the microcontroller proposal

\section{Results}

The first feasibility studies of the two proposals concluded that a microcontroller proposal will be developed. The main reason for choosing this option was due to the components being more modern due to the rapid evolution of the electronics and informatics.

Simulated tests were initially performed using Frizzing and AutoCAD software to determine the best arrangement of the components for the device assembly. Next, it was used sets of LEDs (Light Emitting Diode) to simulate the Cherenkov luminosity to perform the sensors calibration.

Tests were performed to verify the device response to the variation of light intensity caused by the blue luminosity inserted in the environment. The tests were conducted recording the device response compared to to a Thermo-Hybrid-Anemometer Portable Digital Lux meter Model THAL-300 made by Instrutherm. This instrument works in the range of 0 to 20000 lux, with resolution of 1 lux and accuracy of $\pm 5 \%$ of reading \pm 8 digits.

The proposed device will use an algorithm specially developed to establish a relationship between the brightness intensity of Cherenkov and the power of the nuclear reactor.
The algorithm will be written in $\mathrm{C}++$, a high-level programming language, with slight modifications for the Adriano Uno system.

In the program, the information entries, the conditions and the intervals for the readings, the necessary calculations, information and exit methods will be established. The input information will be obtained from the color and brightness sensors and the output information will be transmitted via Bluetooth. The programming language will be compiled in the Arduino IDE software and will be loaded to the device via a USB cable.

A color sensor will be used to isolate the Cherenkov radiation from other sources of luminosity by limiting the luminosity readings in the presence of blue coloration, characteristic of Cherenkov radiation. The sensor selected for the device was the Adafruit TCS34725 with infrared filter, because of its RGB logic and infrared filter that guarantee greater accuracy in readings with lower noise.

A luminosity sensor will be responsible for determining the intensity of the Cherenkov radiation, with values in lux. The TSL2561 Adafruit sensor was considered the most suitable for this device, because it is a digital device that accurately captures values between 0.1 and 40,000 lux, and because of the three precise reading channels.

The main output information of the device is the power of the nuclear reactor that will be calculated after processing of input information and application of the necessary mathematical calculation. The information will be transmitted to a screen, an Android device or a web page through the Bluetooth module HC-06, which has been selected for its reliability and speed of data transmission.

Initially, scale tests will be carried out to assemble and calibrate the device, using LED sets to simulate the Cherenkov luminosity. After the results reach satisfactory levels, the validation in the Triga IPR-R1 reactor will be requested, where the final adjustments in the brightness conversion equations for power will be made.

In Figure 9 is shown photograph of the assembled components prior to being placed in the box. 


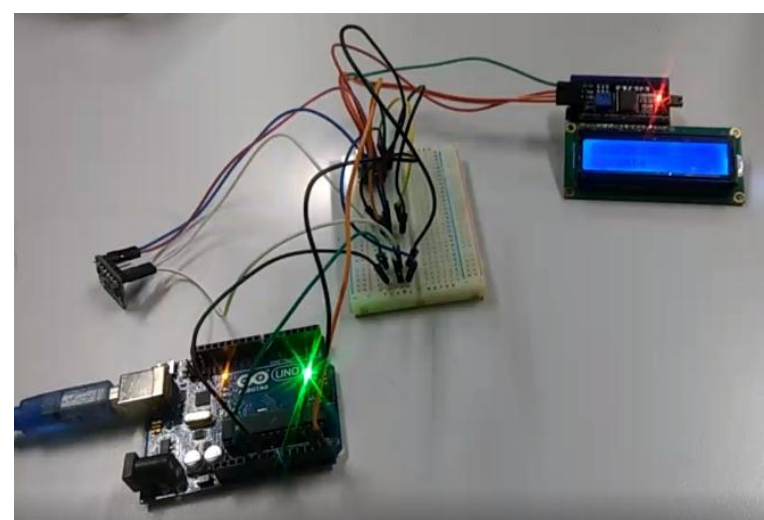

Figure 9 Assembly of components
The initial tests consisted of attaching an opaque blue acrylic plate near the sensors and focusing light with a flashlight on the other side, varying the beam intensity. The experiments were carried out with natural ambient lighting to simulate the operation of the IPR-R1 Trig reactor, varying the distance between the sensor and the light source, starting one centimetre and increasing one centimetre per step. All tests were performed in triplicate, to validate the values obtained. The mean values of the readings are shown in Table 1.

Table 1 Intensity of light obtained experimentally

\begin{tabular}{|c|c|c|c|c|c|c|}
\hline \multirow{4}{*}{$\begin{array}{l} \\
\begin{array}{l}\text { Distance } \\
\text { (cm) }\end{array}\end{array}$} & \multirow{2}{*}{\multicolumn{3}{|c|}{$\begin{array}{l}\text { Prototype } \\
\text { Measure (lux) }\end{array}$}} & \multicolumn{3}{|c|}{ Luximeter } \\
\hline & & & & \multicolumn{3}{|c|}{ Measure (lux) } \\
\hline & \multicolumn{3}{|c|}{ Intensity } & \multicolumn{3}{|c|}{ Intensity } \\
\hline & Low & Mean & High & Low & Mean & High \\
\hline 1 & 44 & 70 & 97 & 43 & 71 & 96 \\
\hline 2 & 32 & 51 & 71 & 32 & 52 & 72 \\
\hline 3 & 27 & 47 & 63 & 28 & 46 & 62 \\
\hline 4 & 18 & 30 & 42 & 18 & 30 & 41 \\
\hline 5 & 13 & 22 & 32 & 14 & 22 & 32 \\
\hline 6 & 11 & 12 & 21 & 10 & 13 & 22 \\
\hline 7 & 7 & 11 & 14 & 7 & 11 & 15 \\
\hline 8 & 4 & 7 & 13 & 5 & 8 & 12 \\
\hline 9 & 5 & 7 & 9 & 4 & 6 & 9 \\
\hline 10 & 2 & 6 & 6 & 3 & 5 & 7 \\
\hline 11 & 1 & 5 & 5 & 2 & 4 & 5 \\
\hline 12 & 3 & 5 & 4 & 2 & 4 & 4 \\
\hline 13 & 2 & 3 & 2 & 2 & 3 & 3 \\
\hline 14 & 3 & 3 & 4 & 2 & 2 & 3 \\
\hline 15 & 0 & 0 & 3 & 1 & 1 & 2 \\
\hline 16 & 0 & 0 & 0 & 0 & 0 & 1 \\
\hline 17 & 0 & 0 & 0 & 0 & 0 & 0 \\
\hline
\end{tabular}

In the tests carried out up to $15 \mathrm{~cm}$ away from the sensors, the readings of the device were compatible with those performed by lux meter Model THAL300 . This was calibrated with ambient light, to record only the variations caused by the insertion of the bluish beam. However, above this distance there was much interference from the ambient light, making it impossible to read.

\section{Conclusions}

Power monitoring channels play a major role in retaining a safe reliable operation of nuclear power reactors and research reactors. Accurate power monitoring using advanced developed channels could make nuclear reactors a more reliable energy source and change public mind about this major energy resource. New generations of nuclear power plants are much safer than their predecessors because of their new accurate safety systems, and more reliable monitoring channels. They produce energy from nuclear fission and are the cleanest, safe and environment-friendly source of energy among many investigated power resources [13].

The power of the core is fundamental for accurate knowledge of the neutron flux, necessary for irradiation experiments and for the estimation of fuel consumption. The precise estimate of burning is important to decrease the quantity of fission products, as well as the activity of the fuel element, the generation of decay heat energy and radio toxicity.

As noted by the IAEA, a greater number of channels to measure power provides a more reliable and safe operation of the reactor [14]. For research reactors, the proposed device may be considered an auxiliary 
measuring tool, increasing redundancy and diversity and providing stable and reliable readings for midrange reactors' central power, improving reactor operation and adding interlocking layers of security.

The advantages of the proposed device are that it will be installed far from the core, making maintenance and adjustment easier than with conventional methods of power measurement. Furthermore, it is a low-cost device, without consumables, easily allowing modifications to improve accuracy and reliability.

\section{Acknowledgment}

The authors of this work wish to thank the following institutions that supported this work: the Nuclear Technology Development Center (CDTN), Brazilian Nuclear Energy Commission (Cnen), Research Support Foundation of the State of Minas Gerais (Fapemig), the Brazilian Council for Scientific and Technological Development (CNPq), and Newton Paiva University Center.

\section{Conflicts of interest}

The authors have no conflicts of interest to declare.

\section{References}

[1] IAEA - International Atomic Energy Agency. Safety of Research Reactors. Safety Standards Series No. SSR-3, Vienna. 2016

[2] IAEA - International Atomic Energy Agency. Safety of Nuclear Power Plants: Design, Safety Standards Series No. SSR-2/1. Vienna. 2016.

[3] Mesquita AZ, Rezende HC, Santos AA, Silva VV. Development of methods for monitoring and controlling power in nuclear reactors. RETERMThermal Engineering. 2018; 13(1):24-7.

[4] https://nucleus.iaea.org/RRDB/RR/ReactorSearch.asp x. Accessed 4 February 2018.

[5] http://www.ga.com/triga- advantages. Accessed 4 February 2018.

[6] Arkani M, Gharib M. Reactor core power measurement using Cherenkov radiation and its application in Tehran research reactor. Annals of Nuclear Energy. 2009; 36(7):896-900.

[7] Bentoumi G, Benson B, Chan PK, Gaudet M, Li G, Li $\mathrm{L}$, et al. Reactor power monitoring using Cherenkov radiation transmitted through a small-bore metallic tube. Annals of Nuclear Energy. 2018; 114:86-91.

[8] http://large.stanford.edu/courses/2014/ph241/alaeian2/ . Accessed 16 August 2018.
[9] https://www.hamamatsu.com/blobs/1328779697620?b lobheadername $1=$ content-

disposition\&blobheadervalue $1=$ inline $\% 3$ Bfilename $\% 3$ D1328482676720.jpg\&ssbinary=t rue. Accessed 4 July 2018.

[10] http://www.meccanismocomplesso.org/wpcontent/uploads/2014/06/ArduinoUno_R3_Front.jpg. Accessed 4 February 2018.

[11] http://s3.amazonaws.com/img.iluria.com/product/152 BD1/3174AD/450xN.jpg. Accessed 4 February 2018.

[12] http://mekhos.com.br/mekhos/wp-con tent/uploads/2017/04/GY-31-Color-Sensor-usingTCS3200.jpg. Accessed 7 July 2018.

[13] Hashemi-Tilehnoee M, Javidkia F. Improving the performance of the power monitoring channel. In Nuclear Reactors 2012

[14] IAEA - International Atomic Energy Agency. Operational Limits and Conditions and Operating Procedures for Research Reactors. DS261 Draft Safety Guide, Vienna. 2006.

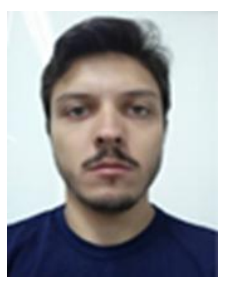

Brício Mares Salles Master in progress in Science and Technology of Nuclear Reactors. Specialist in Industrial Management. MBA in Team Management and Strategic Leadership. Bachelor in Chemical Engineering. Technical Manager and Project Mentor at FabLAB Newton, Brazil.

Email: briciomares@hotmail.com

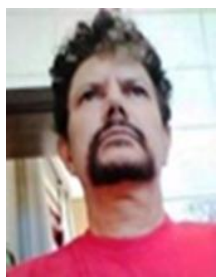

Amir Zacarias Mesquita is a Doctor of Science in Chemical Engineering, Unicamp, Brazil (2005), Master of Science and Nuclear Technologies UFMG/Brazil (1981); Graduated in Electrical Engineering - UFMG/Brazil (1978). He is a researcher, nuclear reactor senior operator, and postgraduate professor. He is involved in research in nuclear engineering with an emphasis on reactor technology, thermo-fluid dynamics and reactor physics.

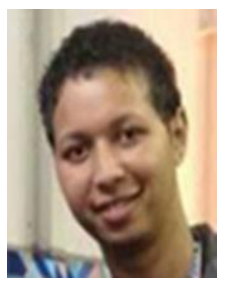

Marley Rosa Luciano is a Trainee of Management and Planning at CreaMG/Brazil. Previous: Faculty of Engineering of Sorocaba (Facens), and Newton Paiva University Center. He studied Engineering of Control and Automation in the Newton Paiva University Center. Previous: CetelTechnological Center for Electroelectronics César Rodrigues-Senai-MG and EE Professor Moraiso. 